\title{
Role of everolimus in the treatment of renal cell carcinoma
}

This article was published in the following Dove Press journal:

Therapeutics and Clinical Risk Management

26 August 2009

Number of times this article has been viewed

\author{
Saby George' \\ Ronald M Bukowski \\ 'University of Texas Health Sciences \\ Center, MC-822I, Division \\ of Hematology and Oncology, \\ San Antonio, Texas, USA; \\ ${ }^{2} \mathrm{CCF}$ Lerner College of Medicine \\ Division of Hematology and Oncology, \\ Cleveland, Ohio, USA
}

\begin{abstract}
The therapeutic options in metastatic renal cell carcinoma have been recently expanded by the discovery of the $V H L$ gene, the mutation of which is associated with development of clear cell carcinoma, and overexpression of the angiogenesis pathway, resulting in a very vascular tumor. This breakthrough in science led to the development of a variety of small molecules inhibiting the VEGF-dependent angiogenic pathway, such as sunitinib and sorafenib. These agents prolong overall and progression-free survival, respectively. The result was the development of robust front-line therapies which ultimately fail and are associated with disease progression. In this setting, there existed an unmet need for developing second-line therapies for patients with refractory metastatic renal cell carcinoma (MRCC). Everolimus (RAD 001) is an oral inhibitor of the mammalian target of rapamycin (mTOR) pathway. The double-blind, randomized, placebo-controlled phase III trial of everolimus (RECORD-1) conducted in MRCC patients after progression on sunitinib or sorafenib, or both, demonstrated a progression-free survival benefit favoring the study drug (4.9 months vs 1.9 months, HR 0.33 , 95\% CI 0.25 to $0.43, P \leq 00.001)$. Everolimus thus established itself as a standard of care in the second-line setting for patients with MRCC who have failed treatment with VEGF receptor inhibitors.
\end{abstract}

Keywords: mTOR inhibitor, mammalian target of rapamycin inhibitor, signal transduction inhibitor, renal cell carcinoma, targeted therapy

\section{Introduction}

Renal cell carcinoma (RCC) is a very aggressive malignancy originating in the renal cortex. Annual incidence in the US is approximately 54,000 and 13,000 patients die as a result. ${ }^{1}$ More than $90 \%$ of renal tumors are RCC and $85 \%$ of RCC are clear cell type. The majority (more than $85 \%$ ) of sporadic RCC is a result of the VHL gene mutation (locus 3p25). ${ }^{2}$ Familial VHL syndrome accounts for a small number of RCC. VHL mutation results in the activation of the angiogenesis pathway that, in turn, makes the tumor very vascular and dependent on this pathway for survival and proliferation. ${ }^{2}$

The discovery of the $V H L$ gene and its significance in RCC led to drug development based on inhibition of the angiogenic pathway, which in turn led to the introduction of various targeted therapies in this disease. Prior to 2005, the standard of care for metastatic renal cell carcinoma (MRCC) patients was immunotherapy including interferon (IFN) and interleukin., ${ }^{3,4}$ The phase II randomized discontinuation trial (RDT) of sorafenib, a tyrosine kinase inhibitor (TKI), in MRCC led to establishment of sorafenib as a viable option in RCC patients. ${ }^{5}$ The randomized phase III double-blinded trial (TARGETS) of sorafenib vs placebo in the cytokine refractory setting demonstrated a doubling of progression-free survival (PFS) favoring sorafenib (5.5 vs 2.8 months;
Correspondence: Saby George University of Texas Health Sciences Center, MC-822I, Division of Hematology and Oncology 7979 Wurzbach Rd, San Antonio, TX-78229, USA

Tel + I 210-450-1667

Fax +1 210-450-1666

Email georges3@uthscsa.edu 
hazard ratio $[\mathrm{HR}]-0.44 ; 95 \%$ confidence interval [CI] 0.35 to $0.55 ; P \leq 0.01) .{ }^{6}$ The phase III trial of sunitinib, another small-molecule TKI of the vascular endothelial growth factor (VEGF) and other pathways, showed robustness in prolonging overall survival (OS) (26.4 vs 21.4 months; HR -0.821 ; $95 \%$ CI 0.673 to $1.001 ; P=0.051)$ and PFS (11 vs 5.5 months; HR $-0.42 ; 95 \%$ CI 0.32 to $0.54 ; P<0.001$ ) when compared head to head with interferon in the first-line setting of MRCC. ${ }^{7}$ These results were clinically meaningful and demonstrated the effect on OS favoring sunitinib. CALGB-90206 and the AVOREN trial demonstrated the efficacy of combining bevacizumab with IFN which produces a PFS in the range of 8.5 to 10.2 months. ${ }^{8} 9$ A randomized phase III trial of temsirolimus vs IFN vs combination of both (Global ARCC) demonstrated the survival benefit in MRCC patients 10.9 vs 7.3 vs 8.4 months (HR for death $0.73 ; 95 \%$ CI 0.58 to 0.92 ; $P=0.008) .{ }^{10}$

The above trials established the new standard of care in MRCC which included small-molecule TKIs such as sunitinib, sorafenib, combination of bevacizumab with IFN, and the mTOR (mammalian target of rapamycin inhibitor) inhibitor temsirolimus. ${ }^{6-10}$ These new treatments produce a variable frequency of tumor regression, with prolongation of PFS and OS, but ultimately the tumor becomes refractory to therapy by various mechanisms. Utilization of an alternative agent such as the mTOR inhibitor everolimus in TKI-refractory RCC is therefore reasonable. There is no established molecular or biological evidence of this drug working preferentially in tumors which are refractory to a TKI, but clearly the use of an agent with an alternative mechanism of action was a reasonable strategy.

\section{Molecular targets and mechanism of action of everolimus}

Everolimus is an orally available inhibitor of mTOR, an intra-cytoplasmic serine-threonine kinase which recognizes stress response signals in cancer via the PI3K-AKT pathway. ${ }^{11}$ This signal transduction inhibition prevents the downstream signaling involved in survival and proliferation of tumor cells. The consequences of mTOR signaling include phosphorylation of p70 ribosomal S6K1 along with the eukaryotic initiation factor $4 \mathrm{E}$ binding protein-1 (4EBP1). The phosphorylation of 4EBP1 results in the release of elf-4E, which in turn allows the cap-dependent translation of proteins. The mTOR kinase also has some control over the angiogenic pathway through the hypoxiainducible factor $1 \mathrm{alfa}$ (HIF1 $\alpha$ ) and VEGF and is linked to endothelial proliferation. ${ }^{12,13}$ Thus the inhibition or signal blockade of the mTOR kinase will in turn result in the cutoff of signals from the stress response signals, prevention of protein translation in cancer cells and also VEGF-dependent angiogenic pathway. In theory this is a multi-prong attack against RCC.

\section{Clinical trials of everolimus in RCC}

Everolimus has been investigated in RCC as a single agent as well as in combination with other potent molecules.

\section{Everolimus monotherapy}

\section{Phase I data}

The phase I trial of RAD 001 was conducted by O'Donnell et al in a variety of advanced solid tumors. This study aimed at reaching the optimal regimen and dosage of everolimus. ${ }^{11}$ Patients were treated in 2-part trial. The first part evaluated the daily dose of everolimus at 5, 10 and $15 \mathrm{mg}$ per day along with the toxicity, antitumor activity, pharmacokinetics and the dose-response relationship to S6K1 activity in peripheral blood mononuclear cells (PBMCs). The study was complemented by blood and tumor drug levels and S6K1 activity study in tumor bearing rats. Part II of the study evaluated the weekly doses of $50 \mathrm{mg}$ and $70 \mathrm{mg}$ and the respective pharmacokinetics and pharmacodynamics.

A total of 92 patients were treated in the phase I trial. Eighteen patients were treated in the weekly regimen in part 1. In part II, 37 patients on a weekly schedule and 37 patients on a daily schedule were treated. In part I, there was no dose limiting toxicity (DLT) reported. The S6K1 activity was suppressed 24 hours after the fourth dose in the PBMC and the duration of suppression lengthened with higher dosage. The daily dosing was able to demonstrate more sustained inhibition of S6K1 as opposed to the weekly regimen.

In part II, DLT occurred in 1 out of the 6 patients who were in the $50 \mathrm{mg}$ weekly group and none of the 4 patients in the $70 \mathrm{mg}$ weekly group. DLT was not observed in 4 patients who were taking $5 \mathrm{mg}$ daily, but in 1 out of the 6 patients who were on $10 \mathrm{mg}$ daily dosing. Thus the higherdose cohorts were expanded in the weekly and daily regimen. The common drug-related adverse events (AE) included fatigue (34\%), rash (48\%) and gastrointestinal toxicities (66\%). There were 5 serious adverse events resulting in 4 hospitalizations and 3 patients required discontinuation of the therapy. No drug-related mortality was reported by the investigators. The elimination half-life was estimated to be around $30 \pm 8$ hours, which was similar to the healthy controls. RECIST evaluation demonstrated 4 partial responses (PR) and 5 of the $10 \mathrm{RCC}$ patients were progression 
free at 6 months. The maximum tolerated dose (MTD) for everolimus was not reached in this trial.

\section{Pharmacokinetics/pharmacodynamics}

Everolimus is an orally bioavailable inhibitor of the mTOR pathway which is metabolized by the CYP3A4 system. The PBMC-derived S6K1 suppression was noticed at 24 hours in all dose levels after the 4th dose. The length of sustained S6K1 suppression was longer with higher doses. The minimum dose that would produce a minimum of 7 days inhibition of S6K1 was $20 \mathrm{mg} .{ }^{11}$

Pharmacokinetic profile was studied in 26 out of 31 patients in the weekly regimen group and 10 patients in the daily regimen. In the weekly dose group, steady state was achieved by the 2 nd week and there was only minimal accumulation. It was also observed that $\mathrm{C}_{\max }$ increased in a dose-dependent manner at doses $5 \mathrm{mg}$ to $20 \mathrm{mg}$, but was less dose proportional at higher doses. The elimination half-life was $30 \pm 8$ hours at all dose levels.

In the daily dosing group, the steady state of the drug was reached within a week and peak concentrations achieved within 1 hour in all but 1 patient. The drug level in serum and area under the curve (AUC) increased in a dose-dependent fashion. The AUC was predicted by the steady state trough levels in addition to plasma concentrations and sustained inhibition of $\mathrm{S} 6 \mathrm{~K} 1$ noticed at $\geq 20 \mathrm{~g}$ in the weekly group and $\geq 5 \mathrm{mg}$ in the daily group. This correlated well with the antitumor activity seen in preclinical models.

\section{Phase II data}

The above results were followed by 2 phase II studies of everolimus in renal cell carcinoma. The first phase II study was conducted by Jac et al in metastatic RCC patients. ${ }^{14}$ The eligibility criteria included predominant clear cell histology, measurable disease, adequate organ function, absence of central nervous system metastasis, Zubrod performance status (ZPS) of 2 or better and no more than 1 prior treatment. The therapy was RAD- 001 given at $10 \mathrm{mg}$ daily dose with no interruptions on a 28-day cycle. PET-CT was also utilized in addition to RECIST defined criteria for response-evaluation which was performed at the end of each cycle for 2 cycles. Forty-one patients were enrolled and 37 were evaluable for toxicity and response. Median age of the cohort was 60 years and 31 were male. Thirty-one patients had received prior therapy for MRCC and the ZPS was 0 in 23 patients, 1 in 13 patients and 2 in 3 patients. There were 12 PR (32\%), 19 (51\%) had stable disease (SD) for $>3$ months and the rest progressed. Median duration of treatment was 8 months and median OS was 11.5 months. Most common treatment-related toxicities included mucositis, skin rash, pneumonitis, hypophosphatemia, hyperglycemia, hypertriglyceridemia and hypercholesterolemia. There was a reduction in the FDG uptake in patients who had a response or stable disease.

The second study was conducted in patients who had failed either sunitinib or sorafenib and had not received $>2$ prior regimens. ${ }^{15}$ The primary end point was response rate to everolimus utilized in the daily dosing schedule of $10 \mathrm{mg}$ orally. The eligibility criteria included presence of clear cell carcinoma, measurable disease, adequate organ function, absence of central nervous system metastasis and good performance status. Therapy was administered with everolimus at $10 \mathrm{mg}$ daily with no treatment breaks on a 28-day cycle. RECIST criteria were used for evaluating the response at the end of each 2 cycles. Twenty-two patients were treated and majority were male (67\%), the median age of the cohort was 57 years and all patients had ZPS 0 or 1 . Three patients withdrew from the study prior to 2 cycles and were excluded from the response evaluation. The best response was $3 \mathrm{PR}$ (16\%), 14 (74\%) SD that lasted for $\geq 3$ months and the rest progressed. The median PFS was 5.5 months and the OS was beyond 8 months. Commonly seen treatment related adverse events included (grade 1 to 2) hypertriglyceridemia (73\%), hyperglycemia (59\%), hypercholesterolemia (64\%), stomatitis (45\%), rash (32\%), nausea (27\%) and diarrhea (18\%). Mostly seen grade 3-4 adverse event was pneumonitis (27\%). This phase II trial demonstrated everolimus as a viable agent and its potential in the second-line setting in RCC patients.

\section{Phase III trial of everolimus in metastatic RCC}

The promising results of everolimus in the TKI-refractory setting of RCC led to the double-blind randomized placebo controlled phase III trial of everolimus in metastatic RCC patients who progressed on VEGF-targeted therapy. ${ }^{16}$ This multi-center trial was conducted at 86 centers in Australia, Canada, Europe, Japan, and the USA. The patients had metastatic RCC with clear cell component in the tumor and had progressed on sunitinib or sorafenib or both. Prior treatments allowed also included interferon, interleukin, chemotherapy, bevacizumab and radiotherapy. More than $95 \%$ of the patients in the treatment arm and placebo arm had undergone nephrectomy prior to enrolment in this study. The eligibility criteria included presence of measurable disease, Karnofsky Performance status of $\geq 70 \%$ and adequate solid organ and marrow function. 
Exclusion criteria included prior exposure to mTOR inhibitors (temsirolimus), untreated brain metastasis or uncontrolled co-morbidities. Eligible patients were randomized 2:1 to everolimus vs placebo. Patient stratification was based on the Memorial Sloan Kettering Cancer Center (MSKCC) prognostic scoring system and also the previous anti-RCC therapy (one vs 2 VEGF receptor tyrosine kinase inhibitor). ${ }^{17}$ The treatment included continuous dosing of oral everolimus at $10 \mathrm{mg}$ daily or placebo in addition to best supportive care. A treatment cycle was defined as 28 days of treatment and safety evaluation was done every 2 weeks for 3 cycles and at the end of each cycle after the third cycle.

Treatment in both cohorts continued until progression, severe toxicity, death or discontinuation. Randomization was revealed only at progression and cross-over to open-label everolimus was permissible upon progression on placebo. Blinded independent central review was utilized for RECIST response evaluation. ${ }^{18}$ PFS was the primary end-point; the time from randomization to the earliest sign of progression or death. Secondary endpoints were safety, objective response rate (ORR), OS, disease-related symptoms and quality of life (QOL).

Efficacy was assessed on an intent-to-treat basis after randomization. All patients who received a dose of everolimus and followed up were assessed for safety. QOL was assessed utilizing the European Organization for Research and Treatment of Cancer (EORTC) QLQ-C30 and Functional Assessment of Cancer Therapy Kidney Symptom Index-Disease-Related Symptoms (FKSI-DRS) questionnaires. $^{19,20}$

The sample size was calculated based on the prolongation of PFS by $50 \%$ from 3 months to 4.5 months and a risk-reduction of $33 \%$ (corresponding to a HR of 0.67 ). The 3-month PFS for placebo was assessed based on data from the TARGET trial where the placebo treated arm had a PFS of 2.8 months after failing prior cytokine based therapy. ${ }^{6}$ One sided cumulative $\alpha$ of 0.025 in the 2:1 random allocation calculated the need for 290 events so as to achieve a power of $90 \%$ in this study. Interim analyses were planned at $30 \%$ and $60 \%$ of the total (290) events and the final analysis at 290 events. The interim analysis allowed the study to be discontinued for reasons of safety, futility, or lack of efficacy. Lan DeMets and O'Brien-Fleming spending functions were utilized with pre-defined stopping boundaries. The patients who did not progress or die at the time of data cutoff were censored. Kaplan-Meier method was used for PFS and OS estimation.
After screening 554 patients, 410 were randomly assigned in a 2:1 ratio to everolimus or placebo. There were 272 patients in the everolimus arm and 138 in the placebo arm. The median age in everolimus arm was 61 and 60 in the placebo arm. Nearly $71 \%$ in the everolimus group and $79 \%$ in the placebo group had progressed during prior therapy and the median length of treatment was 95 days in everolimus and 57 days in placebo respectively. Majority (96\% in everolimus and $95 \%$ in placebo) had undergone prior nephrectomy. Nearly two-third of patients in each arm had $\geq 3$ sites of disease. The reasons for discontinuation of treatment included death, progressive disease, adverse events and withdrawal of consent by the patient. At the time of data cutoff, the PFS in everolimus assessed by the independent central reviewers was significantly longer than placebo arm $(\mathrm{HR}=0.33 ; 95 \%$ CI 0.25 to $0.43 ; P \leq 0.001)$. The best responses observed in the two arms were $1 \%$ vs $0 \%$ PR, $63 \%$ vs $32 \%$ SD, $19 \%$ vs $46 \% \mathrm{PD}$ favoring everolimus. In $17 \%$ patients on everolimus and $22 \%$ patients on placebo, disease could not be assessed. Median PFS was 4.9 (4 to 5.5 months) months vs 1.9 months (1.8 to 1.9 months) favoring everolimus. At 6 months, progression-free probability was $26 \%$ in patients receiving everolimus and $2 \%$ in patients receiving placebo.

Pre-defined exploratory and subset analyses demonstrated benefit from everolimus in all groups based on age, sex, prior VEGF-targeted therapy and geographical region. The median OS has not been reached at the time of the data cutoff (Table 1). The overall survival data from this trial is still being awaited.

No observable difference was evident between the two groups with respect to the time to clear-cut deterioration of patient-reported outcomes. The evaluation of the FKSI-DRS risk score and the EORTC QLQ-C30 score indicated that the QOL was sustained in the everolimus group compared to placebo. The above scoring system compared various factors including physical functioning, global health status/ quality of life, role functioning, emotional functioning, cognitive functioning, social functioning and symptoms. The above observation favoring the everolimus group was irrespective of the increased adverse events seen in the active treatment arm.

Adverse events were more frequent in everolimus compared to placebo and the most commonly reported AE in the study drug arm included stomatitis (44\%), rash $(29 \%)$, fatigue $(31 \%)$, asthenia (33\%), anemia $(92 \%)$, hypercholesterolemia $(77 \%)$, hypertriglyceridemia $(73 \%)$ and hyperglycemia $(57 \%){ }^{21}$ The most commonly seen grade 3 AEs in the everolimus arm included stomatitis 
Table I Demographics of the patients included in the phase III trial of everolimus ${ }^{16}$

\begin{tabular}{llll}
\hline Factor & Hazard ratio & P-value & Number \\
\hline Central review & 0.30 & $<0.001$ & 410 \\
Investigator review & 0.31 & $<0.001$ & 410 \\
MSKCC & & & \\
$\quad$ Favorable & 0.35 & $<0.001$ & 118 \\
$\quad$ Intermediate & 0.25 & $<0.001$ & 231 \\
$\quad$ Poor & 0.39 & 0.009 & 61 \\
Prior therapy & & & \\
$\quad$ Sorafenib & 0.29 & $<0.001$ & 119 \\
Sunitinib & 0.30 & $<0.001$ & 184 \\
$\quad$ Both & 0.28 & $<0.001$ & 107 \\
Age & & & \\
$\quad<65$ & 0.32 & $<0.001$ & 259 \\
$\quad>65$ & 0.29 & $<0.001$ & 151 \\
Sex & & & \\
Male & 0.29 & $<0.001$ & 317 \\
Female & 0.36 & 0.002 & 93 \\
Region & & & \\
USA and Canada & 0.24 & $<0.001$ & 130 \\
Europe & 0.37 & 0.001 & 251 \\
Japan and Australia & 0.10 & & \\
\hline
\end{tabular}

Abbreviation: MSKCC, Memorial Sloan Kettering Cancer Center.

(4\%), fatigue $(5 \%)$, pneumonitis $(4 \%)$, anemia $(12 \%)$, hypercholesterolemia (4\%), hyperglycemia (15\%), lymphopenia (16\%) and hypophosphatemia $(6 \%)$ (Table 2$){ }^{21}$ There were 22 patients who developed non-infectious pneumonitis in the everolimus arm compared to none in the placebo arm. ${ }^{16}$ Six out of the 8 patients who developed grade 3 pneumonitis had to discontinue the everolimus therapy. ${ }^{16}$ This is a serious adverse event that is defined as the

Table 2 Table demonstrating commonly seen grade 3 or 4 toxicities (update from the package insert) ${ }^{21}$

\begin{tabular}{|c|c|c|c|c|}
\hline \multirow[t]{3}{*}{ Adverse events } & \multicolumn{2}{|c|}{ Everolimus $(\mathbf{N}=\mathbf{2 7 4})$} & \multicolumn{2}{|c|}{ Placebo $(\mathbf{N}=137)$} \\
\hline & Grade 3 & Grade 4 & Grade 3 & Grade 4 \\
\hline & $\%$ & $\%$ & $\%$ & $\%$ \\
\hline Stomatitis & 4 & $<1$ & 0 & 0 \\
\hline Fatigue & 5 & 0 & 3 & $<1$ \\
\hline Infections & 7 & 3 & I & 0 \\
\hline Pneumonitis & 4 & 0 & 0 & 0 \\
\hline Anemia & 12 & 1 & 5 & $<1$ \\
\hline Hypercholesterolemia & 4 & 0 & 0 & 0 \\
\hline Hyperglycemia & 15 & $<1$ & 1 & 0 \\
\hline Lymphopenia & 16 & 2 & 5 & 0 \\
\hline Hypophosphatemia & 6 & 0 & 0 & 0 \\
\hline
\end{tabular}

radiographic lung change irrespective of signs or symptoms (pleural effusion, hypoxia, cough, dyspnea, malaise), in the absence of a non-drug cause. Further studies on this adverse event are under way. ${ }^{16}$

The objective response rate was $1 \%$ in the everolimus arm and none in the placebo arm. Even though everolimus was not associated with impressive objective responses, the prolongation of PFS demonstrated by this drug was definitely a correlation with the increased number of SD observed (Table 3). This is an interesting phenomenon, where RECIST-defined tumor shrinkage is not necessary for PFS advantage. This is an indirect indication of why RECIST-defined tumor evaluation is probably not the best way to assess the response to the signal transduction inhibitors, including everolimus.

\section{Everolimus combinations in RCC}

The combination of everolimus with another agent is a viable option if the second agent targets the signaling in a different pathway. This phase II study by Whorf et al utilized the combination of bevacizumab with everolimus and enrolled patients with advanced RCC into two groups. ${ }^{22}$ Bevacizumab was dosed at $10 \mathrm{mg} / \mathrm{kg}$ iv every 2 weeks and everolimus was used at $10 \mathrm{mg}$ orally daily until progression. Patients with advanced clear cell RCC or metastatic or recurrent RCC, with ECOG performance status of 1 or better were enrolled in this study. There were two groups of patients based on prior treatment: A, no prior treatment or B, prior sunitinib or sorafenib. Treatment evaluation was done using the RECIST criteria at the end of every 8 weeks. ${ }^{18}$

Fifty-nine patients (30 to group A and 29 to group B) were enrolled. The median age was 65 years with $76 \%$ having intermediate Motzer prognostic score and $73 \%$ with prior nephrectomy. There were 42 patients who received therapy for at least 8 weeks, 6 patients were non-evaluable and 11 were not at the 8 -week mark. The best response in the evaluable patients $(n=48)$ was $21 \%$ ORR and $69 \% \mathrm{SD} /$ minor response. Toxicities included proteinuria (19\% grade $3-4)$, fatigue (9\% grade $3-4$ ) mucositis/stomatitis ( $49 \%$ grade $3-4)$, hyperlipidemia ( $45 \%$ grade $3-4$ ), nausea ( $40 \%$ grade $3-4$ ) and hypertension $(25 \%)$. The final results of this study are pending. The interim results are suggestive of the feasibility and relative safety of the combination of everolimus and bevacizumab.

Report of a phase I trial combining sunitinib and everolimus was reported at the 2009 ASCO Annual meeting. ${ }^{23}$ Cohorts of patients were enrolled to receive sunitinib at $37.5 \mathrm{mg}$ in the 4 weeks on/2 weeks off schedule along with RAD001 in a daily or weekly regimen. ${ }^{23}$ The combination produced DLT in cohort 4 (sunitinib at $37.5 \mathrm{mg}$ and RAD001 
Table 3 Response and outcome ${ }^{16}$

\begin{tabular}{lll}
\hline & $\begin{array}{l}\text { Everolimus } \\
(\mathbf{N}=\mathbf{2 7 2})\end{array}$ & $\begin{array}{l}\text { Placebo } \\
\mathbf{( N = 1 3 8 )}\end{array}$ \\
\hline $\begin{array}{l}\text { Progression-free survival } \\
\text { No. of progression events }\end{array}$ & I0I (37\%) & $90(65 \%)$ \\
$\begin{array}{l}\text { Censored } \\
\text { I7I (63\%) }\end{array}$ & $48(35 \%)$ \\
$\begin{array}{l}\text { Best objective response } \\
\text { Partial response }\end{array}$ & $3(1 \%)$ & 0 \\
$\quad$ Stable disease & $171(63 \%)$ & $44(32 \%)$ \\
Progressive disease & $53(19 \%)$ & $63(46 \%)$ \\
$\quad$ Could not be assessed & $45(17 \%)$ & $31(22 \%)$ \\
Overall deaths & $42(15 \%)$ & $26(19 \%)$ \\
\hline
\end{tabular}

at $30 \mathrm{mg}$ weekly). The severe adverse events included endocarditis, gastrointestinal hemorrhage with severe anemia and pulmonary embolism. The recommended dose for phase II trial of this combination is sunitinib at $37.5 \mathrm{mg}$ in a 4 weeks on 2 weeks off fashion with everolimus at $20 \mathrm{mg}$ weekly. ${ }^{23}$

\section{Ongoing clinical trials of everolimus}

After the efficacy and PFS benefit from everolimus in RCC was established by the RECORD-1 trial, a series of phase I and II clinical trials have been started for evaluating the benefit of combining everolimus with various other active agents (Table 4). There are trials evaluating the efficacy of everolimus in non-clear cell carcinoma and also papillary histology.

\section{Conclusion}

Everolimus is a potent orally available mTOR inhibitor, which has shown to be beneficial in the second-line setting of metastatic RCC. Everolimus was associated with several adverse events, both metabolic and systemic, but was tolerated reasonably well by the majority of patients. Everolimus produces few RECIST type responses, however, tumor shrinkage analysis demonstrates over $70 \%$ of patients had some degree of shrinkage, and that mostly stable disease is produced in this population of refractory patients. The high rate of SD translated into a PFS advantage which is double that of placebo. This phenomenon may also be partially due to the fact that the patients had received more than one line of therapy (TKI, cytokines and other therapies) prior to trial enrolment and, tumors were refractory to different lines of therapy. This is similar to the phenomenon seen with sorafenib in the TARGETS trial, where patients were treated in the second line, and the response rate to sorafenib was $\leq 10 \%$, but there was PFS and survival advantage. The patients who had prior sunitinib $(n=124)$ had better PFS compared to the placebo $(\mathrm{n}=60)(3.88$ months vs 1.84 months; HR 0.34 ; $95 \%$ CI 0.23 to $0.51 ; P<0.001)$. Similarly the patients who had prior sorafenib $(n=81)$ also did much better than placebo $(n=43)(5.88$ months vs 2.83 months; HR $0.25 ; 95 \%$ CI 0.16 to $0.42 ; P<0.001)$. The above established everolimus as a choice for treating patients with RCC after they fail sunitinib or sorafenib. ${ }^{24}$

The phase III trial of everolimus was conducted after the approval of sorafenib, sunitinib and also temsirolimus for patients with RCC. In this scenario the trial was performed in the TKI refractory setting, which represented an unmet clinical need. The FDA approved everolimus in metastatic

Table $4 \mathrm{~A}$ list of ongoing trials obtained from Clinicaltrials.gov

\begin{tabular}{|c|c|c|}
\hline Combination/phase & Clinical trial identifier & Center/Sponsor \\
\hline Everolimus and sunitinib/phase lb & NCT00788060 & Duke University, NC, USA \\
\hline Everolimus and sorafenib/phase I/II & NCT00384969 & $\begin{array}{l}\text { Univ. of California, } \\
\text { San Francisco, CA, USA }\end{array}$ \\
\hline $\begin{array}{l}\text { Bevacizumab + everolimus vs } \\
\text { bevacizumab + IFN/randomized } \\
\text { phase II RECORD } 2\end{array}$ & NCT007I9264 & $\begin{array}{l}\text { International multi-center } \\
\text { trial by Novartis and } \\
\text { Hoffmann-La Roche }\end{array}$ \\
\hline $\begin{array}{l}\text { Bevacizumab and everolimus in } \\
\text { treatment refractory RCC/phase II }\end{array}$ & NCT0065I482 & Stanford University, CA, USA \\
\hline $\begin{array}{l}\text { Everolimus and sorafenib } \\
\text { combination/phase I/II }\end{array}$ & NCT0039282I & $\begin{array}{l}\text { Sarah Cannon Research } \\
\text { Institute, USA }\end{array}$ \\
\hline Everolimus and vatalanib/phase I & NCT00655655 & Mayo Clinic, MN, USA \\
\hline $\begin{array}{l}\text { Exploratory study evaluating } \\
\text { fluorodeoxyglucose - Position }\end{array}$ & NCT00529802 & University of Chicago, IL, USA \\
\hline Emission Tomography as a Predictive & & \\
\hline $\begin{array}{l}\text { Marker for Therapy with everolimus } \\
\text { in Metastatic Renal Cell Cancer }\end{array}$ & & \\
\hline
\end{tabular}

Abbreviations: IFN, interferon; RCC, renal cell carcinoma. 
RCC patients who are refractory to sunitinib or sorafenib. The increased number of SD and PFS advantage seen in the everolimus arm proved that RECIST-defined tumor shrinkage is probably not necessary for PFS benefit. It is also demonstrating an unmet need for functional imaging studies in RCC patients treated with signal transduction inhibitors like everolimus, and thus changing the standard of evaluation from the traditional RECIST evaluation to a more meaningful evaluation that would correlate with the activity of the tumors. Per the RECIST evaluation this could be further characterized as high disease stabilization effect, as in the case of sorafenib. The changes observed in the FDG PET CT after cycle 2 (phase II trial) in patients who had a PR or SD could mean that it is more of a meaningful way of evaluating response to drugs that block signaling. This could also mean that the RECIST defined SD may indeed be contributing to the potential progression-free survival benefit from this agent. But the above needs to be validated by larger studies. The revised RECIST (ver 1.1) also allows the judicious use of FDG PET-CT to complement CT findings. ${ }^{25}$

The adverse events were more frequent in the everolimus arm compared to the placebo group. The adverse events were unique in the sense that there were metabolic adverse events like hyperlipidemia, hyperglycemia, hypophosphatemia etc in addition to the class-specific non-infectious pneumonitis.

In addition, the QOL of advanced RCC patients treated with everolimus was maintained, independent of the adverse events produced by everolimus. This means that everolimus may be a good choice in patients with advanced disease where the goals could be re-set to improvement in the quality of life and disease stabilization with PFS benefit rather than tumor shrinkage. This drug is in the same class as temsirolimus, but its advantage is that it is orally dosed opposed to the weekly iv dosing of temsirolimus, which could be cumbersome. For the above reasons, everolimus was approved in advanced RCC patients, who failed prior TKI-based therapy.

Everolimus is an orally bioavailable agent, whereas temsirolimus is an intravenous prodrug. Both agents are mTOR inhibitors, differences relate to their pharmacodynamic and pharmacokinetic properties. The differences in their indications in RCC relate to study design and patient populations in which the respective trials were conducted.

There should also be front-line trials of everolimus in advanced RCC as it has proven to be an effective second-line agent. This drug might bring forth more responses, if tried in the front-line setting. The activity of everolimus in the front-line setting may be much better than in the TKI-1 setting, due to the difference in biology of the disease between treatment-naive and TKI-refractory settings. The various combination trials of everolimus with agents which work in pathways other than mTOR signaling would be worth waiting for. Phase IV studies of everolimus for assessing the safety in large populations are underway.

\section{Disclosures}

The authors declare no conflicts of interest.

\section{References}

1. Jemal A, Siegel R, Ward E, et al. Cancer statistics. CA Cancer J Clin. 2008;58:71-96.

2. George DJ, Kaelin WG Jr. The von Hippel-Lindau protein, vascular endothelial growth factor, and kidney cancer. $N$ Engl J Med. 2003;349:419-421.

3. McDermott DF, Regan MM, Clark JI, et al. Randomized phase III trial of high-dose interleukin-2 versus subcutaneous interleukin-2 and interferon in patients with metastatic renal cell carcinoma. J Clin Oncol. 2005;23:133-141.

4. De Mulder PH, Oosterhof G, Bouffioux C, et al. EORTC (30885) randomised phase III study with recombinant interferon alpha and recombinant interferon alpha and gamma in patients with advanced renal cell carcinoma. The EORTC Genitourinary Group. Br J Cancer. 1995;71:371-375.

5. Ratain MJ, Eisen T, Stadler WM, et al. Phase II placebo-controlled randomized discontinuation trial of sorafenib in patients with metastatic renal cell carcinoma. J Clin Oncol. 2006;24:2505-2512.

6. Escudier B, Eisen T, Stadler WM, et al. Sorafenib in advanced clear-cell renal-cell carcinoma. $N$ Engl J Med. 2007;356:125-134.

7. Motzer RJ, Hutson TE, Tomczak P, et al. Sunitinib versus interferon alfa in metastatic renal-cell carcinoma. $N$ Engl J Med. 2007;356:115-124.

8. Escudier B, Pluzanska A, Koralewski P, et al. Bevacizumab plus interferon alfa-2a for treatment of metastatic renal cell carcinoma: a randomised, double-blind phase III trial. Lancet. 2007;370:2103-2111.

9. Rini BI, Halabi S, Rosenberg JE, et al. Bevacizumab plus interferon alfa compared with interferon alfa monotherapy in patients with metastatic renal cell carcinoma: CALGB 90206. J Clin Oncol. 2008;26: 5422-5428.

10. Hudes G, Carducci M, Tomczak P, et al. Temsirolimus, interferon alfa, or both for advanced renal-cell carcinoma. $N$ Engl J Med. 2007;356:2271-2281.

11. O'Donnell A, Faivre S, Burris HA 3rd, et al. Phase I pharmacokinetic and pharmacodynamic study of the oral mammalian target of rapamycin inhibitor everolimus in patients with advanced solid tumors. J Clin Oncol. 2008;26:1588-1595.

12. Corradetti MN, Guan KL. Upstream of the mammalian target of rapamycin: do all roads pass through mTOR? Oncogene. 2006;25: 6347-6360.

13. Mamane Y, Petroulakis E, LeBacquer O, et al. mTOR, translation initiation and cancer. Oncogene. 2006;25:6416-6422.

14. Jac J. A phase II trial of RAD001 in patients (Pts) with metastatic renal cell carcinoma (MRCC). J Clin Oncol. ASCO, 2007;261s: Abstract 5107.

15. Jac J. A phase II study with a daily regimen of the oral mTOR inhibitor RAD001 (everolimus) in patients with metastatic renal cell carcinoma which has progressed on tyrosine kinase inhibition therapy. J Clin Oncol. ASCO, May 2008;20 Suppl:Abstract 5113.

16. Motzer RJ, Escudier B, Oudard S, et al. Efficacy of everolimus in advanced renal cell carcinoma: a double-blind, randomised, placebocontrolled phase III trial. Lancet. 2008;372:449-456.

17. Motzer RJ, Bacik J, Mazumdar M. Prognostic factors for survival of patients with stage IV renal cell carcinoma: Memorial Sloan-Kettering Cancer Center experience. Clin Cancer Res. 2004;10:6302S-6303S. 
18. Therasse P. Evaluation of response: new and standard criteria. Ann Oncol. 2002;13 Suppl 4:127-129.

19. Aaronson NK, Ahmedzai S, Bergman B, et al. The European Organization for Research and Treatment of Cancer QLQ-C30: a quality-of-life instrument for use in international clinical trials in oncology. J Natl Cancer Inst. 1993;85:365-376.

20. Cella D, Yount S, Brucker PS, et al. Development and validation of a scale to measure disease-related symptoms of kidney cancer. Value Health. 2007;10:285-293.

21. Afinitor (Everolimus) [package insert] March, 2009.

22. Whorf RC HJ, Spigel DR, et al. Phase II study of bevacizumab and everolimus (RAD001) in the treatment of advanced renal cell carcinoma (RCC). J Clin Oncol. ASCO, 2008;252s:Abstract 5010.
23. Kroog GS. Phase I trial of RAD001 (everolimus) plus sunitinib in patients with metastatic renal cell carcinoma. J Clin Oncol. 2009;27(15S): Abstract 5037.

24. Sternberg C. Everolimus (RAD001) vs placebo after VEGFR-TKI failure in metastatic renal cell carcinoma, European Society for Medical Oncology; 2008. http://www.esmo.org/fileadmin/media/ presentations/977/2035/RAD001\%20Sternberg\%20ESMO\%201409 2008FINAL.ppt.pdf.

25. Eisenhauer EA, Therasse P, Bogaerts J, et al. New response evaluation criteria in solid tumours: revised RECIST guideline (version 1.1). Eur J Cancer. 2009;45:228-247.

\section{Publish your work in this journal}

Therapeutics and Clinical Risk Management is an international, peerreviewed journal of clinical therapeutics and risk management, focusing on concise rapid reporting of clinical studies in all therapeutic areas, outcomes, safety, and programs for the effective, safe, and sustained use of medicines. This journal is indexed on PubMed Central, CAS,
EMBase, Scopus and the Elsevier Bibliographic databases. The manuscript management system is completely online and includes a very quick and fair peer-review system, which is all easy to use. Visit http://www.dovepress.com/testimonials.php to read real quotes from published authors.

Submit your manuscript here: http://www.dovepress.com/therapeutics-and-clinical-risk-management-journal 